\title{
On a Subclass of P-Valent Functions Defined by a Generalized Salagean Operator
}

\author{
Ozokeraha Christiana Funmilayo \\ Department of Statistics, Delta State Polytechnic, Oghara, Nigeria
}

Email address:

andchrisben@gmail.com

To cite this article:

Ozokeraha Christiana Funmilayo. On a Subclass of P-valent Functions Defined by a Generalized Salagean Operator by. Pure and Applied Mathematics Journal. Vol. 7, No. 4, 2018, pp. 45-62. doi: 10.11648/j.pamj.20180704.11

Received: July 31, 2018; Accepted: September 18, 2018; Published: November 6, 2018

\begin{abstract}
In recent times, the study of analytic functions has been useful in solving many problems in mechanics, Laplace equation, electrostatics, etc. An analytic function is said to be univalent in a domain if it does not take the same value twice in that domain while an analytic function is said to be p-valent in a domain if it does not take the same value more than $\mathrm{p}$ times in that domain. Many researches on properties of p-valent functions using Salagean, Al Oboudi and Opoola differential operators have been reviewed. The aim of this research is to obtain the properties of new subclasses of p-valent functions defined by Salagean differential operator and its objectives are to obtain new subclasses of p-valent functions and the necessary properties for the new subclasses. This research will be a contribution to knowledge in geometric function theory and provide new tools of applications in fluid dynamics and differential equations. This paper introduces new subclasses of $p-$ valent functions defined by $\mathrm{Al}$-Oboudi differential operator. Finally, the paper studies some interesting results including subordination, coefficient inequalities, starlikeness and convexity conditions, Hadamard product and certain properties of neighbourhoods of the new subclasses of $\mathrm{p}$-valent functions. Theorems were used to establish certain conditions of the new subclasses of $\mathrm{p}$-valent functions.
\end{abstract}

Keywords: P-Valent Functions, Analytic Functions, Differential Operator, Subordination

\section{Introduction}

Let

$$
f(z)=z+\sum_{k=2}^{\infty} a_{k} z^{k} \quad z \in U
$$

where $U=\{z:|z|<1\}$

The Salagean differential $D^{n}$ operator for univalent functions is defined as

$$
\begin{gathered}
D^{0} f(z)=f(z) \\
D^{1} f(z)=z f^{\prime}(z)
\end{gathered}
$$

and

$$
D^{n} f(z)=D\left\{D^{n-1} f(z)\right\} \quad(n \in N)
$$

with

$$
D^{n} f(z)=z+\sum_{k=2}^{\infty} k^{n} a_{k} z^{k} \quad(n \in N)
$$

The Al Oboudi differential operator for univalent functions is defined as

$$
\begin{gathered}
D^{0} f(z)=f(z) \\
D^{1} f(z)=\lambda z f^{\prime}(z)+(1-\lambda) f(z) \\
D_{\lambda}^{n} f(z)=D_{\lambda}^{n}\left\{D_{\lambda}^{n-1} f(z)\right\}
\end{gathered}
$$

and 


$$
D_{\lambda}^{n} f(z)=z+\sum_{k=2}^{\infty}[1+(k-1) \lambda]^{n} a_{k} z^{k} \quad n \in N, \quad \lambda \geq 0
$$

The Opoola differential operator for univalent functions is defined as

$$
\begin{gathered}
D^{0}(\mu, \beta, t) f(z)=f(z) \\
D^{1}(\mu, \beta, t) f(z)=t z f^{\prime}(z)-z(\mu-\beta) t+[1+(\beta-\mu-1) t] f(z) \\
D_{t}^{n} f(z)=D_{t}^{n}\left\{D_{t}^{n-1} f(z)\right\}
\end{gathered}
$$

and

$$
D_{t}^{n}(\mu, \beta, t) f(z)=z+\sum_{k=2}^{\infty}[1+(k+\beta-\mu-1) t]^{n} a_{k} z^{k} \quad n \in N_{0} \quad 0 \leq \mu \leq \beta, \text { (12) } \quad t \geq 0
$$

Definition 1.1

A function $f(z)$ is called a p-valent function if it does not take any value more than $\mathrm{p}$ times

$$
f^{\prime}(z)=p z^{p-1}+\sum_{k=1}^{\infty}(p+k) a_{p+k} z^{p+k-1}
$$

Let $A(p)$ be the class of functions $f(z)$ of the form

$$
f(z)=z^{p}+\sum_{k=1}^{\infty} a_{p+k} z^{p+k} \quad\{p \in N=1,2,3 \ldots\}, \quad z \in U
$$

$$
\begin{gathered}
z f^{\prime}(z)=z\left(p z^{p-1}+\sum_{k=1}^{\infty}(p+k) a_{p+k} z^{p+k-1}\right) \\
z f^{\prime}(z)=p z^{p}+\sum_{k=1}^{\infty}(p+k) a_{p+k} z^{p+k}
\end{gathered}
$$

Where

$$
U=\{z:|z|<1\} \quad(n \in N)
$$

When

$$
\begin{gathered}
n=1 \\
D^{1} f(z)=p z^{p}+\sum_{k=1}^{\infty}(p+k) a_{p+k} z^{p+k}
\end{gathered}
$$

The Salagean differential $D^{n}$ operator for p-valently functions is defined as

$$
\begin{aligned}
& D^{0} f(z)=f(z) \\
& D^{1} f(z)=z f^{\prime}(z)
\end{aligned}
$$

and

Note:

$$
D^{n} f(z)=D\left\{D^{n-1} f(z)\right\} \quad(n \in N)
$$

with

$$
D^{n} f(z)=p^{n} z^{p}+\sum_{k=1}^{\infty}(p+k)^{n} a_{p+k} z^{p+k}(p \varepsilon N=1,2,3, \ldots) \quad(n \in N)
$$

[15]

Let $A(p)$ denote the class of analytic functions defined in the unit disc $U=\{z:|z|<1\}$ of the form

$$
f(z)=z^{p}+\sum_{j=1}^{\infty} a_{p+j} z^{p+j} \quad\{p \in N=1,2,3 \ldots\}, \quad z \in U
$$

Some properties of the subclass of $A(p)$ were studied by Aouf et al.

$T(p)$ denote a subclass of $A(p)$ consisting of functions $f(z)$ of the form

$$
f(z)=z^{p}-\sum_{j=1}^{\infty}\left|a_{p+j}\right| z^{p+j}
$$

We denote by $T(p, \alpha), C(p, \alpha), 0 \leq a<p$ of the class of starlike functions of order $\alpha$ and the class of convex functions of order $\alpha$ respectively, where 


$$
T^{*}(p, \alpha)=\left\{f \in T^{*}(p): \operatorname{Re}\left\{\frac{z f^{\prime}(z)}{f(z)}\right\}>\alpha, z \in u\right\}
$$

And

$$
C(p, \alpha)=\left\{f \in T^{*}(p): \operatorname{Re}\left\{1+\frac{z f^{\prime \prime}(z)}{f^{\prime}(z)}\right\}>\alpha, z \in u\right\}
$$

Note that $T^{*}(p, \alpha) \subset T(p)$ and $C(p, \alpha) \subset T(p)$.

Sezgin et al [19] studied a class of p-valent functions with negative coefficients using Salagean derivatives and coefficients estimates, distortion theorems and extreme points for this class was given.

$$
\begin{aligned}
& \text { Let } f(z)=z^{p}+\sum_{j=1}^{\infty} a_{p+j} z^{p+j} \text { if } \\
& f(z)=z^{p}+\sum_{j=1}^{\infty}\left(a_{p+j}\right)^{n}(\eta+j)\left|a_{p+j}\right| \leq \eta p^{n}
\end{aligned}
$$
then

$$
f \in S_{n}(p, \eta)
$$

Also

If $f \in T_{n}(p, \eta)$ then

$$
r^{p}-\frac{\eta p^{n}}{(1+\eta)(p+1)^{n}} z^{p+1} \leq\left|f^{\prime}(z)\right| \leq r^{p}+\frac{\eta p^{n}}{(1+\eta)(p+1)^{n}} r^{p+1}(|z|=r)
$$

With equality for

$$
f(z)=z^{p}-\frac{\eta p^{n}}{(1+\eta)(p+1)^{n}} z^{p+1}(z= \pm r)
$$

Lastly

$$
f_{p}(z)=z^{p} \text { and } f_{p+j}(z)=z^{p}-\frac{\eta p^{n}}{(1+\eta)(p+1)^{n}} z^{p+j}(j=1,2, \ldots)
$$

Then $f \in T_{n}(p, \eta)$ if and only if it can be expressed in the form

$$
f(z)=z^{p}+\sum_{j=0}^{\infty} \lambda_{p+j} f_{p+j}(z) \text { where } \lambda_{p+j} \geq 0 \text { and } \sum_{j=0}^{\infty} \lambda_{p+j}=1 \text { (See [19]) }
$$

Dileep and Latha derived characterization theorem and radius of starlikeness for certain class of p-valent analytic functions. Noor et al [15] introduced a subclass of p-valent functions involving Chokwon-Srivastava integral and studied some interesting results including inclusion relation, convolution with convex function and integral preserving property for the class.

Let $A_{p}$ denote the class of functions of the form

$$
f(z)=z^{p}+\sum_{k=1}^{\infty} a_{p+k} z^{p+k} \quad\{p \in N=1,2,3 \ldots\}
$$

which are p-valent in the open unit disc $E=\{z \in C:|z|<1\}$.

The operator $Q_{p}(a, c)$ is defined as follows

$$
Q_{p}(a, c ; z)=\sum_{k=0}^{\infty} \frac{(a)_{k}}{(c)_{k}} z^{p+k}, a \in R, c \in R \backslash Z_{0}^{-}, Z_{0}^{-}=\{0 .-1, \ldots\}, z \in E
$$

Where $(x)_{k}$ is the shifted factorial defined in terms of gamma function by

$$
(x)_{k}=\frac{\Gamma(x+k)}{\Gamma(x)}=\left\{\begin{array}{cc}
1 & k=0, x \in C \backslash\{0\} \\
x(x+1) \ldots(x+k-1) & k \in N, x \in C
\end{array}\right\}
$$

Using convolution, the operator $L_{p}(a, c)$ can be defined as 


$$
L_{p}(a, c) f(z)=Q_{p}(a, c ; z) * f(z)=z^{p}+\sum_{k=1}^{\infty} \frac{(a)_{k}}{(c)_{k}} z^{p+k}, z \in E
$$

Where $a \in R, c \in R \backslash Z_{0}^{-}, f \in A_{p}$ and $Q_{p}(a, c)$ is defined in (24). For details, see [13, 14].

The purpose of this research is to study some properties of p-valent functions defined by the Al-Oboudi differential operator.

\section{Main Results}

\subsection{AlOboudi Differential Operator forP-Valently Functions}

Let $A(p)$ be the class of functions $f(z)$ of the form

$$
f(z)=z^{p}+\sum_{k=1}^{\infty} a_{p+k} z^{p+k} \quad\{p \in N=1,2,3 \ldots\}, \quad z \in U
$$

where $U=\{z:|z|<1\}$

$$
D_{\lambda}^{n} f(z)=p^{n} z^{p}+\sum_{k=1}^{\infty}[1+(p+k-1) \lambda]^{n} a_{p+k} z^{p+k} \quad(n \in N) \quad \lambda \geq 0
$$

When $n=1$

$$
\begin{gathered}
D^{1} f(z)=p z^{p}+\sum_{k=1}^{\infty}[1+(p+k-1) \lambda] a_{p+k} z^{p+k} \\
\left.D^{1} f(z)=p z^{p}+\sum_{k=1}^{\infty} a_{p+k} z^{p+k}+\lambda \sum_{k=1}^{\infty}(p+k)\right] a_{p+k} z^{p+k}-\lambda \sum_{k=1}^{\infty} a_{p+k} z^{p+k} \\
D^{1} f(z)=p z^{p}+z^{p}+\sum_{k=1}^{\infty} a_{p+k} z^{p+k}-\lambda z^{p}-\lambda \sum_{k=1}^{\infty} a_{p+k} z^{p+k}+\lambda p z^{p}+\lambda \sum_{k=1}^{\infty}(p+k) a_{p+k} z^{p+k}-\lambda p z^{p}-z^{p}+\lambda z^{p} \\
D^{1} f(z)=z^{p}+\sum_{k=1}^{\infty} a_{p+k} z^{p+k}-\lambda\left[z^{p}+\sum_{k=1}^{\infty} a_{p+k} z^{p+k}\right]+\lambda z\left(p z^{p-1}+\sum_{k=1}^{\infty}(p+k) a_{p+k} z^{p+k-1}\right)+p z^{p}-\lambda p z^{p}-z^{p}+\lambda z^{p} \\
D^{1} f(z)=f(z)-\lambda f(z)+\lambda z f^{\prime}(z)+z^{p}(p-\lambda p-1+\lambda) D^{1} f(z)=(1-\lambda) f(z)+\lambda z f^{\prime}(z)+z^{p}(p-\lambda p-1+\lambda)
\end{gathered}
$$

When $\quad p=1$

$$
\begin{gathered}
D^{1} f(z)=(1-\lambda) f(z)+\lambda z f^{\prime}(z)+z^{1}(1-\lambda(1)-1+\lambda) \\
D^{1} f(z)=(1-\lambda) f(z)+\lambda z f^{\prime}(z)+z(1-\lambda-1+\lambda) \\
D^{1} f(z)=(1-\lambda) f(z)+\lambda z f^{\prime}(z)+0 \\
D_{\lambda}^{1} f(z)=(1-\lambda) f(z)+\lambda z f^{\prime}(z)
\end{gathered}
$$

Note:

$$
\left.\lambda p z^{p}+\lambda \sum_{k=1}^{\infty}(p+k) a_{p+k} z^{p+k}=\lambda z\left[p z^{p-1}+\sum_{k=1}^{\infty}(p+k)\right] a_{p+k} z^{p+k-1}\right]
$$




$$
\begin{gathered}
f^{\prime}(z)=p z^{p-1}+\sum_{k=1}^{\infty}(p+k) a_{p+k} z^{p+k-1} \\
z f^{\prime}(z)=z\left(p z^{p-1}+\sum_{k=1}^{\infty}(p+k) a_{p+k} z^{p+k-1}\right) \\
z f^{\prime}(z)=p z^{p}+\sum_{k=1}^{\infty}(p+k) a_{p+k} z^{p+k}
\end{gathered}
$$

The Al Oboudi differential operator for p-valently functions is defined as

$$
\begin{gathered}
D^{0} f(z)=f(z) \\
D^{1} f(z)=\lambda z f^{\prime}(z)+(1-\lambda) f(z)
\end{gathered}
$$

and

$$
D_{\lambda}^{n} f(z)=p^{n} z^{p}+\sum_{k=1}^{\infty}[1+(p+k-1) \lambda]^{n} a_{p+k} z^{p+k}(p \varepsilon N=1,2,3, \ldots) \quad(n \in N) \quad \lambda \geq 0
$$

Definition 2.1

A function $f \in A(p)$ is said to be in the class $A_{P}(n, m, A, B, \lambda)$ if

$$
\frac{D_{\lambda}^{n+m} f(z)}{D_{\lambda}^{n} f(z)} \prec \frac{1+A z}{1+B z} \quad z \in U
$$

Where $n \in N, \quad m \in N, \quad \lambda \geq 0$ and $-1 \leq A<B \leq 1$ with

$$
D_{\lambda}^{n} f(z)=p^{n} z^{p}+\sum_{k=1}^{\infty}[1+(p+k-1) \lambda]^{n} a_{p+k} z^{p+k} \quad n \in N_{0}, \quad \lambda \geq 0
$$

and

$$
D_{\lambda}^{n+m} f(z)=p^{n+m} z^{p}+\sum_{k=1}^{\infty}[1+(p+k-1) \lambda]^{n+m} a_{p+k} z^{p+k} \quad n \in N_{0} \quad m \in N_{0} \quad \lambda \geq 0
$$

Neighbourhoods of certain $p$ valent analytic functions have been considered by several researchers. $([10,11,14,16])$

$$
A_{P}(n, m, A, B, \lambda)
$$

\subsection{Neighbourhood for the Class}

Theorem 2.1

A function $f \in A(p)$ is said to be in the class $A_{P}(n, m, A, B, \lambda)$ if and only if

$$
\begin{gathered}
\sum_{k=1}^{\infty}[1+(p+k-1) \lambda]^{n}\{(1+A)-(1-B) \\
{[1+(p+k-1) \lambda]^{m} a_{p+k} \leq p^{n}\left[B p^{m}-A-1+p^{m}\right]} \\
\text { for } n \in N, \quad m \in N, \quad \lambda \geq 0 \text { and }-1 \leq A<B \leq 1, \quad\{p \in N=1,2,3 \ldots\}
\end{gathered}
$$

Proof - subordination 
Let

$$
\begin{aligned}
& f \in A_{P}(n, m, A, B, \lambda) \text { then } \\
& \frac{D_{\lambda}^{n+m} f(z)}{D_{\lambda}^{n} f(z)} \prec \frac{1+A z}{1+B z} \quad z \in U
\end{aligned}
$$

We need to introduce $w(z)$

That is,

$$
\begin{gathered}
\frac{D_{\lambda}^{n+m} f(z)}{D_{\lambda}^{n} f(z)}=\frac{1+A w(z)}{1+B w(z)} \\
w(0)=0,|w(z)|<1
\end{gathered}
$$

Cross multiplying we have

$$
\begin{gathered}
D_{\lambda}^{n} f(z)[1+A w(z)]=D_{\lambda}^{n+m} f(z)[1+B w(z)] \\
D_{\lambda}^{n} f(z)+A w(z) D_{\lambda}^{n} f(z)=D_{\lambda}^{n+m} f(z)+B w(z) D_{\lambda}^{n+m} f(z) \\
D_{\lambda}^{n} f(z)-D_{\lambda}^{n+m} f(z)=B w(z) D_{\lambda}^{n+m} f(z)-A w(z) D_{\lambda}^{n} f(z) \\
D_{\lambda}^{n} f(z)-D_{\lambda}^{n+m} f(z)=w(z)\left[B D_{\lambda}^{n+m} f(z)-A D_{\lambda}^{n} f(z)\right] \\
w(z)=\frac{D_{\lambda}^{n} f(z)-D_{\lambda}^{n+m} f(z)}{B D_{\lambda}^{n+m} f(z)-A D_{\lambda}^{n} f(z)}
\end{gathered}
$$

Definition 2.2

Let $f(z)$ and $g(z)$ be analytic functions in the disk $\mathrm{U}$. Then we say that $f(z)$ is subordinate to $g(z)$ written $f(z) \prec g(z)$ if there exists an analytic function $w(z)$ such that $w(0)=0,|w(z)|<1, \quad z \in D$ and $f(z)=g(w(z))$. [16]

Hence to prove subordination we have

$$
|w(z)|=\left|\frac{D_{\lambda}^{n} f(z)-D_{\lambda}^{n+m} f(z)}{B D_{\lambda}^{n+m} f(z)-A D_{\lambda}^{n} f(z)}\right|<1
$$

But

$$
D_{\lambda}^{n} f(z)=p^{n} z^{p}+\sum_{k=1}^{\infty}[1+(p+k-1) \lambda]^{n} a_{p+k} z^{p+k} \quad n \in N_{0} \quad \lambda \geq 0
$$

and

$$
D_{t}^{n+m} f(z)=p^{n+m} z^{p}+\sum_{k=1}^{\infty}[1+(p+k-1) \lambda]^{n+m} a_{p+k} z^{p+k} \quad n \in N_{0} \quad m \in N_{0} \quad \lambda \geq 0
$$




$$
\begin{aligned}
& p^{n} z^{p}+\sum_{k=1}^{\infty}\left[(1+(p+k-1) \lambda]^{n} a_{p+k} z^{p+k}-\right. \\
& |w(z)|=\mid \frac{p^{n+m} z^{p}+\sum_{k=1}^{\infty}\left[(1+(p+k-1) \lambda]^{n+m} a_{p+k} z^{p+k}\right.}{B\left[p^{n+m} z^{p}+\sum_{k=1}^{\infty}\left[(1+(p+k-1) \lambda]^{n+m} a_{p+k} z^{p+k}\right]-\right.} k 1 \\
& A\left[p^{n} z^{p}+\sum_{k=1}^{\infty}\left[(1+(p+k-1) \lambda]^{n} a_{p+k} z^{p+k}\right]\right. \\
& p^{n} z^{p}-p^{n+m} z^{p}+\sum_{k=1}^{\infty}\left[(1+(p+k-1) \lambda]^{n} a_{p+k} z^{p+k}\right. \\
& |w(z)|=\left|\frac{-\sum_{k=1}^{\infty}\left[( 1 + ( p + k - 1 ) \lambda ] ^ { n } \left[(1+(p+k-1) \lambda]^{m} a_{p+k} z^{p+k}\right.\right.}{B p^{n+m} z^{p}-A p^{n} z^{p}+B \sum_{k=1}^{\infty}\left[(1+(p+k-1) \lambda]^{n}\left[(1+(p+k-1) \lambda]^{m} a_{p+k} z^{p+k}\right]\right.}\right|<1 \\
& -A \sum_{k=1}^{\infty}\left[(1+(p+k-1) \lambda]^{n} a_{p+k} z^{p+k}\right. \\
& p^{n} z^{p}\left[1-p^{m}\right]+\sum_{k=1}^{\infty}\left[(1+(p+k-1) \lambda]^{n}\{[1-\right. \\
& |w(z)|=\left|\frac{\left.\sum_{k=1}^{\infty}[(1+(p+k-1) \lambda)]^{m}\right\} a_{p+k} z^{p+k}}{p^{n} z^{p}\left[B p^{m}-A\right]+\sum_{k=1}^{\infty}\left[( 1 + ( p + k - 1 ) \lambda ] ^ { n } \left\{\left[B(1+(p+k-1) \lambda]^{m}\right.\right.\right.}\right|<1 \\
& -A]\} a_{p+k} z^{p+k}
\end{aligned}
$$

Thus

$$
\begin{gathered}
p^{n} z^{p}\left[1-p^{m}\right]+\sum_{k=1}^{\infty}\left[(1+(p+k-1) \lambda]^{n}\{[1-\right. \\
\operatorname{Re}\left\{\frac{\sum_{k=1}^{\infty}\left[(1+(p+k-1) \lambda]^{m}\right\} a_{p+k} z^{p+k}}{p^{n} z^{p}\left[B p^{m}-A\right]+\sum_{k=1}^{\infty}\left[( 1 + ( p + k - 1 ) \lambda ] ^ { n } \left\{\left[B(1+(p+k-1) \lambda]^{m}\right.\right.\right.}\right\}<1 \\
-A]\} a_{p+k} z^{p+k}
\end{gathered}
$$

Taking $|z|=r$ for sufficiently small $r$ with $0<r<1$ the denominator of (38) is positive so it is positive for all $r$ with $0<r<1$ since $w(z)$ is analytic for $|z|<1$ then the inequality yields

$$
\begin{aligned}
& p^{n} r^{p}\left[1-p^{m}\right]+\sum_{k=1}^{\infty}\left[( 1 + ( p + k - 1 ) \lambda ] ^ { n } \left\{\left[1-\sum_{k=1}^{\infty}\left[(1+(p+k-1) \lambda]^{m}\right\} a_{p+k} r^{p+k}\right.\right.\right. \\
& <p^{n} r^{p}\left[B p^{m}-A\right]+\sum_{k=1}^{\infty}\left[(1+(p+k-1) \lambda]^{n}\left\{\left[B(1+(p+k-1) \lambda]^{m}-A\right]\right\} a_{p+k} r^{p+k}\right.
\end{aligned}
$$


That is,

$$
\begin{aligned}
& \sum_{k=1}^{\infty}\left[( 1 + ( p + k + \beta - \mu - 1 ) t ] ^ { n } \left\{\left[1-\sum_{k=1}^{\infty}\left[(1+(p+k-1) \lambda]^{m}\right\} a_{p+k} r^{p+k}-\right.\right.\right. \\
& \sum_{k=1}^{\infty}\left[(1+(p+k-1) \lambda]^{n}\left\{\left[B(1+(p+k-1) \lambda]^{m}-A\right]\right\} a_{p+k} r^{p+k}\right. \\
& \leq p^{n} r^{p}\left[B p^{m}-A\right]-p^{n} r^{p}\left[1-p^{m}\right]
\end{aligned}
$$

Equivalently

$$
\begin{aligned}
& \sum_{k=1}^{\infty}\left[( 1 + ( p + k - 1 ) \lambda ] ^ { n } \left\{\left[1-\sum_{k=1}^{\infty}\left[(1+(p+k-1) \lambda]^{m}\right\} a_{p+k} r^{p+k}-\right.\right.\right. \\
& \sum_{k=1}^{\infty}\left[(1+(p+-1) \lambda]^{n}\left\{\left[B(1+(p+k-1) \lambda]^{m}-A\right]\right\} a_{p+k} r^{p+k}\right. \\
& \leq p^{n} r^{p}\left[B p^{m}-A-1+p^{m}\right]
\end{aligned}
$$

And (40) gives

$$
\begin{aligned}
& \sum_{k=1}^{\infty}\left[( 1 + ( p + k - 1 ) \lambda ] ^ { n } \left\{(1+A)-(1+B)\left[(1+(p+k-1) \lambda]^{m}\right\} a_{p+k} r^{p+k}\right.\right. \\
& \leq p^{n} r^{p}\left[B p^{m}-A-1+p^{m}\right]
\end{aligned}
$$

and the theorem 2.1 follows upon letting $r^{p+k}=1=r^{p}$.

That is,

$$
\begin{aligned}
& \sum_{k=1}^{\infty}\left[( 1 + ( p + k - 1 ) \lambda ] ^ { n } \left\{(1+A)-(1+B)\left[(1+(p+k-1) \lambda]^{m}\right\} a_{p+k}\right.\right. \\
& \leq p^{n}\left[B p^{m}-A-1+p^{m}\right]
\end{aligned}
$$

(34) can be written as

$$
\begin{aligned}
& \sum_{k=1}^{\infty}\left[( 1 + ( p + k - 1 ) \lambda ] ^ { n } \left\{(A-B)\left[(1+(p+k-1) \lambda]^{m}\right\} a_{p+k}\right.\right. \\
& \leq p^{n}\left[B p^{m}-A-1+p^{m}\right]
\end{aligned}
$$

This completes the proof of theorem 2.1.

Conversely for $\left|z^{p}\right|=r^{p}, 0<r^{p}<1$, we have

$$
\begin{aligned}
& \sum_{k=1}^{\infty}\left[( 1 + ( p + k - 1 ) \lambda ] ^ { n } \left\{(1+A)-(1+B)\left[(1+(p+k-1) \lambda]^{m}\right\} a_{p+k} r^{p+k}\right.\right. \\
& \leq \sum_{k=1}^{\infty}\left[( 1 + ( p + k - 1 ) \lambda ] ^ { n } \left\{(1+A)-(1+B)\left[(1+(p+k-1) \lambda]^{m}\right\} a_{p+k} r^{p}\right.\right.
\end{aligned}
$$

From theorem 2.1 we have 


$$
\begin{aligned}
& \mid p^{n} z^{p}\left[1-p^{m}\right]+\sum_{k=1}^{\infty}\left[( 1 + ( p + k - 1 ) \lambda ] ^ { n } \left\{\left[1-\sum_{k=1}^{\infty}\left[(1+(p+k-1) \lambda]^{m}\right\} a_{p+k} z^{p+k} \mid\right.\right.\right. \\
& \leq p^{n} r^{p}\left[1-p^{m}\right]+\sum_{k=1}^{\infty}\left[( 1 + ( p + k - 1 ) \lambda ] ^ { n } \left\{\left[1-\sum_{k=1}^{\infty}\left[(1+(p+k-1) \lambda]^{m}\right\} a_{p+k} r^{p+k}\right.\right.\right. \\
& <p^{n} r^{p}\left[B p^{m}-A\right]+\sum_{k=1}^{\infty}\left[(1+(p+k-1) \lambda]^{n}\left\{\left[B(1+(p+k-1) \lambda]^{m}-A\right]\right\} a_{p+k} r^{p+k}\right. \\
& <\mid p^{n} z^{p}\left[B p^{m}-A\right]+\sum_{k=1}^{\infty}\left[(1+(p+k-1) \lambda]^{n}\left\{\left[B(1+(p+k-1) \lambda]^{m}-A\right]\right\} a_{p+k} z^{p+k}\right.
\end{aligned}
$$

This proves that

$$
\frac{D_{t}^{n+m} f(z)}{D_{t}^{n} f(z)} \prec \frac{1+A z}{1+B z} \quad z \in U
$$

And hence $f \in A_{p}(n, m, A, B, \lambda)$

This completes the proof of definition (2.1).

\subsection{Identity Function of Neighbourhood}

Definition 2.3:

For any function $f \in A(p)$ and $\delta>0$ the $(j, \delta)$ - neighborhood of $f$ is defined as.

$$
N_{j, \partial}(f)=\left\{g(z)=z^{p}+\sum_{k=1}^{\infty} b_{p+k} z^{p+k} \in A(p): \sum_{k=1}^{\infty} k\left|a_{p+k}-b_{p+k}\right| \leq \delta\right\}
$$

Specifically, for the identity function $g(z)=z$,

$$
N_{j, \partial}(e)=\left\{g(z)=z^{p}-\sum_{k=1}^{\infty} b_{p+k} z^{p+k} \in A(p): \sum_{k=1}^{\infty} k\left|b_{p+k}\right| \leq \delta\right\} \quad[16]
$$

Theorem 2.2

If

$$
\delta=\frac{p^{n}\left[B p^{m}-A-1+p^{m}\right]}{[1+p) \lambda]^{n-1}[(A-B)[1+P) \lambda]^{m}}
$$

Note that (41) was used to assume (45) with $\mathrm{k}=1$.

Then

$$
A_{p}(n, m, A, B, \lambda) \subset N_{j, \delta}(e)[16]
$$

Proof

It follows from theorem (2.1) that if

$$
f \in A_{p}(n, m, A, B, \lambda)
$$

Then

$$
\begin{aligned}
& [1+p) \lambda]^{n-1}[(A-B)[1+p) \lambda]^{m} \sum_{k=1}^{\infty} k a_{p+k} \\
& \leq p^{n}\left[B p^{m}-A-1+p^{m}\right]
\end{aligned}
$$


Which implies

$$
\sum_{k=1}^{\infty} k a_{p+k} \leq \frac{p^{n}\left[B p^{m}-A-1+p^{m}\right]}{[1+p) \lambda]^{n-1}[(A-B)[1+p) \lambda]^{m}}=\delta
$$

From definition (2.3) the result follows.

\section{Coefficient Inequalities}

Let $C_{p}$ denote the subclass of $A_{P}(n, m, A, B, \lambda)$ consisting of functions of the form

$$
f(z)=z^{p}-\sum_{k=1}^{\infty} a_{P+k} z^{P+k}\left(a_{P+k} \geq 0\right)
$$

Then

$$
D_{\lambda, p}^{n} f(z)=z^{p}-\sum_{k=1}^{\infty}[1+(p+k-1) \lambda]^{n} a_{k} z^{k} \quad\{p \in N=1,2,3, \ldots\} \quad n \in N_{0} \quad \lambda \geq 0
$$

Definition 3.1

A function $f \in C_{p}$ is said to be in the class $R_{p}^{n}(\alpha, \beta, \gamma, \mu)$ ifand only if

$$
\left|\frac{\left(D_{\lambda, p}^{n} f(z)\right)^{\prime}-p z^{p-1}}{\alpha\left[D_{\lambda, p}^{n} f(z)\right]^{\prime}+(\beta-\gamma)}\right|<\mu \quad(z \in \mu) \quad\left(n \in N_{0}\right)
$$

For $0 \leq \alpha<1, \quad 0 \leq \gamma<1, \quad 0<\beta \leq 1, \quad 0<\mu<1$ where

$$
\begin{aligned}
& D_{\lambda, p}^{n} f(z)=z^{p}-\sum_{k=1}^{\infty}[1+(p+k-1) \lambda]^{n} a_{p+k} z^{p+k} \\
& {\left[D_{\lambda, p}^{n} f(z)\right]^{\prime}=p z^{p-1}-\sum_{k=1}^{\infty}[1+(p+k-1) \lambda]^{n} a_{p+k} z^{p+k-1}} \\
& \left|\frac{p z^{p-1}-\sum_{k=1}^{\infty}[1+(p+k-1) \lambda]^{n} a_{p+k} z^{p+k-1}-p z^{p-1}}{\alpha\left[p z^{p-1}-\sum_{k=1}^{\infty}[1+(p+k-1) \lambda]^{n} a_{p+k} z^{p+k-1}\right]+(\beta-\gamma)}\right|<\mu \\
& \left|\frac{\sum_{k=1}^{\infty}(p+k)[1+(p+k-1) \lambda]^{n} a_{p+k} z^{p+k-1}}{\alpha\left[p z^{p-1}-\sum_{k=1}^{\infty}(p+k)[1+(p+k-1) \lambda]^{n} a_{p+k} z^{p+k-1}\right]+(\beta-\gamma)}\right|<\mu
\end{aligned}
$$

Theorem 3.1

A function $f \in C_{p}$ is said to be in the class $R_{p}^{n}(\alpha, \beta, \gamma, \mu)$ if and only if

$$
\sum_{k=1}^{\infty}(p+k)[1+(p+k-1) \lambda]^{n}(1+\mu \alpha) a_{p+k} \leq \mu(\alpha p+\beta-\gamma)
$$

The result is sharp for the function $\mathrm{f}$ given by 


$$
f(z)=z^{p}-\frac{\mu(\alpha p+\beta-\gamma)}{(p+k)[1+(p+k-1)]^{n}(1+\mu \alpha)} z^{p+k} \quad k \geq 1
$$

Proof

Suppose that $f \in R_{p}^{n}(\alpha, \beta, \gamma, \mu)$ then from (42) we have

$$
\begin{gathered}
\left|\frac{\sum_{k=1}^{\infty}(p+k)[1+(p+k-1) \lambda]^{n} a_{p+k} z^{p+k-1}}{\alpha\left[p z^{p-1}-\sum_{k=1}^{\infty}(p+k)[1+(p+k-1) \lambda]^{n} a_{p+k} z^{p+k-1}\right]+(\beta-\gamma)}\right|<\mu \text { So we obtain } \\
\operatorname{Re}\left\{\frac{\sum_{k=1}^{\infty}(p+k)[1+(p+k-1) \lambda]^{n} a_{p+k} z^{p+k-1}}{\alpha\left[p z^{p-1}-\sum_{k=1}^{\infty}(p+k)[1+(p+k-1) \lambda]^{n} a_{p+k} z^{p+k-1}\right]+(\beta-\gamma)}\right\}<\mu \\
\sum_{k=1}^{\infty}(p+k)[1+(p+k-1) \lambda]^{n} a_{p+k} z^{p+k-1}<\mu\left\{\alpha\left[p z^{p-1}-\sum_{k=1}^{\infty}(p+k)[1+(p+k-1) \lambda]^{n} a_{p+k} z^{p+k-1}\right]+(\beta-\gamma)\right\} \\
\left.\sum_{k=1}^{\infty}(p+k)[1+(p+k-1)]^{n} a_{p+k} z^{p+k-1}<\mu \alpha\left[p z^{p-1}-\sum_{k=1}^{\infty}(p+k)[1+(p+k-1) \lambda]^{n} a_{p+k} z^{p+k-1}\right]+\mu(\beta-\gamma)\right\} \\
\left.\sum_{k=1}^{\infty}(p+k)[1+(p+k-1) \lambda]^{n} a_{p+k} z^{p+k-1}+\mu \alpha\left[\sum_{k=1}^{\infty}(p+k)[1+(p+k-1) \lambda]^{n} a_{p+k} z^{p+k-1}\right]<\mu \alpha\left[p z^{p-1}\right]+\mu(\beta-\gamma)\right\} \\
\sum_{k=1}^{\infty}(p+k)[1+(p+k-1) \lambda]^{n} a_{p+k} z^{p+k-1}(1+\mu \alpha)<\mu \alpha p z^{p-1}+\mu \beta-\mu \gamma \\
\sum_{k=1}^{\infty}(p+k)[1+(p+k-1) \lambda]^{n} a_{p+k} z^{p+k-1}(1+\mu \alpha)<\mu\left(\alpha p z^{p-1}+\beta-\gamma\right)
\end{gathered}
$$

If we choose $\mathrm{z}$ real and according to maximum modulus theorem. Let $z \rightarrow 1^{-}$then we get

$$
\begin{aligned}
& \sum_{k=1}^{\infty}(p+k)[1+(p+k-1) \lambda]^{n} a_{p+k}(1+\mu \alpha)<\mu(\alpha p+\beta-\gamma) \\
& \sum_{k=1}^{\infty}(p+k)[1+(p+k-1) \lambda]^{n} a_{p+k}(1+\mu \alpha)<\mu(\alpha p+\beta-\gamma) \\
& \sum_{k=1}^{\infty}(p+k)[1+(p+k-1) \lambda]^{n} a_{p+k}(1+\mu \alpha)<\mu(\alpha p+\beta-\gamma)
\end{aligned}
$$

Conversely, suppose that the inequality (43) holds true and let $z \in \partial U=\{z \in C:|z|=1\}$

Then we find from (49) that

$$
\left|\left(D_{\lambda, p}^{n} f(z)\right)^{\prime}-p z^{p-1}\right|<\mu\left|\alpha\left[D_{\lambda, p}^{n} f(z)\right]^{\prime}+(\beta-\gamma)\right|
$$




$$
\begin{gathered}
\left|\left(D_{\lambda, p}^{n} f(z)\right)^{\prime}-p z^{p-1}\right|-\mu\left|\alpha\left[D_{\lambda, p}^{n} f(z)\right]^{\prime}+(\beta-\gamma)\right| \\
\left|\sum_{k=1}^{\infty}(p+k)[1+(p+k-1) \lambda]^{n} a_{p+k} z^{p+k-1}\right|-\mu\left|\alpha\left[p z^{p-1}-\sum_{k=1}^{\infty}(p+k)[1+(p+k-1) \lambda]^{n} a_{p+k} z^{p+k-1}\right]+(\beta-\gamma)\right| \\
\leq \sum_{k=1}^{\infty}(p+k)[1+(p+k-1) \lambda]^{n} a_{p+k}|z|^{p+k-1}-\mu \alpha \sum_{k=1}^{\infty}(p+k)[1+(p+k-1) \lambda]^{n} a_{p+k}|z|^{p+k-1}-\mu\left(\alpha p|z|^{p-1}+\beta-\gamma\right) \\
\sum_{k=1}^{\infty}(p+k)[1+(p+k-1) \lambda]^{n}(1+\mu \alpha) a_{p+k}-\mu(\alpha p+\beta-\gamma) \leq 0
\end{gathered}
$$

Hence by the maximum modulus theorem we have $f \in C_{p}^{n}(\alpha, \beta, \gamma, \mu)$

Corollary 3.2: If $f \in C_{p}^{n}(\alpha, \beta, \gamma, \mu)$ then

$$
a_{p+1} \leq \frac{\mu(\alpha p+\beta-\gamma) p^{n}}{(1+p \lambda]^{n}(1+\mu \alpha)}
$$

Proof

From (54) we have

$$
\begin{gathered}
\sum_{k=1}^{\infty}(p+k)[1+(p+k-1) \lambda]^{n}(1+\mu \alpha) a_{k} \leq \mu(\alpha p+\beta-\gamma) \\
a_{p+k}=\frac{\mu(\alpha p+\beta-\gamma)}{\sum_{k=1}^{\infty}(p+k)[1+(p+k-1) \lambda]^{n}(1+\mu \alpha)}
\end{gathered}
$$

But $k=1$

$$
a_{p+1}=\frac{\mu(\alpha p+\beta-\gamma)}{\sum_{k=1}^{\infty}(1)[1+p \lambda]^{n}(1+\mu \alpha)}
$$

So

$$
a_{p+1} \leq \frac{\mu(\alpha p+\beta-\gamma)}{\sum_{k=1}^{\infty}[1+p \lambda]^{n}(1+\mu \alpha)}=\frac{\mu(\alpha p+\beta-\gamma)}{\sum_{k=p+1}^{\infty}[1+p \lambda]^{n}(1+\mu \alpha)}
$$

Theorem 3.3

Let the functions of the form

$$
\begin{array}{r}
f(z)=z^{p}-\sum_{k=1}^{\infty} a_{p+k} z^{p+k} \quad\left(a_{p+k} \geq 0\right) \\
g(z)=z^{p}-\sum_{k=1}^{\infty} b_{p+k} z^{p+k}\left(b_{k} \geq 0\right)
\end{array}
$$

be in the class $R_{p}^{n}(\alpha, \beta, \gamma, \mu)$. Then for $0 \leq \lambda \leq 1$, the function h defined by

$$
h(z)=(1-\lambda) f(z)+\lambda g(z)=z^{p}-\sum_{k=p+1}^{\infty} c_{p+k} z^{p+k}
$$


$c_{p+k}=(1-\lambda) a_{p+k}+\lambda b_{p+k} \geq 0$ is also in the class $R_{p}^{n}(\alpha, \beta, \gamma, \mu)$

Now, note that in theorem 3.3 we have that

$$
\begin{gathered}
h(z)=(1-\lambda) f(z)+\lambda g(z) \\
h(z)=f(z)-\lambda f(z)+\lambda g(z) \\
h(z)=z^{p}-\sum_{k=1}^{\infty} a_{p+k} z^{p+k}-\lambda\left(z^{p}-\sum_{k=1}^{\infty} a_{p+k} z^{p+k}\right)+\lambda\left(z^{p}-\sum_{k=1}^{\infty} b_{p+k} z^{p+k}\right) \\
\left.\left.h(z)=z^{p}-\sum_{k=1}^{\infty} a_{p+k} z^{p+k}-\lambda z^{p}+\lambda \sum_{k=1}^{\infty} a_{p+k} z^{p+k}\right)+\lambda z^{p}-\lambda \sum_{k=p+1}^{\infty} b_{p+k} z^{p+k}\right) \\
\left.\left.h(z)=z^{p}-\sum_{k=1}^{\infty} a_{p+k} z^{k}+\lambda \sum_{k=1}^{\infty} a_{p+k} z^{p+k}\right)-\lambda \sum_{k=1}^{\infty} b_{p+k} z^{p+k}\right) \\
h(z)=z^{p}-\sum_{k=1}^{\infty} z^{p+k}\left(a_{p+k}+\lambda a_{p+k}-\lambda b_{p+k}\right) \\
h(z)=z^{p}-\sum_{k=1}^{\infty} z^{p+k} c_{p+k} \\
c_{p+k}=\left(a_{p+k}+\lambda a_{p+k}-\lambda b_{p+k}\right)
\end{gathered}
$$

$c_{p+k}=(1-\lambda) a_{p+k}+\lambda b_{p+k} \geq 0$ is also in the class $R_{p}^{n}(\alpha, \beta, \gamma, \mu)$

Proof of theorem 3.3

Suppose that each of the functions $\mathrm{f}$ and $\mathrm{g}$ is in the class $R_{p}^{n}(\alpha, \beta, \gamma, \mu)$ then making use of

$$
\sum_{k=1}^{\infty}(p+k)[1+(p+k-1) \lambda]^{n}(1+\mu \alpha) a_{p+k} \leq \mu(\alpha p+\beta-\gamma)
$$

In theorem 3.1weseethatsince $c_{p+k}=(1-\lambda) a_{p+k}+\lambda b_{p+k}$ wehave

$$
\begin{gathered}
\sum_{k=1}^{\infty}(p+k)[1+(p+k-1) \lambda]^{n}(1+\mu \alpha) c_{p+k}=(1-\lambda)\left[\sum_{k=1}^{\infty}(p+k)[1+(p+k-1) \lambda]^{n}(1+\mu \alpha) a_{p+k}+\right. \\
\lambda \sum_{k=1}^{\infty}(p+k)[1+(p+k-1) \lambda]^{n}(1+\mu \alpha) b_{p+k} \\
\leq(1-\lambda) \mu(\alpha p+\beta-\gamma)+\lambda \mu(\alpha p+\beta-\gamma) \\
=\mu(\alpha p+\beta-\gamma)-\lambda \mu(\alpha p+\beta-\gamma)+\lambda \mu(\alpha p+\beta-\gamma) \\
=\mu(\alpha p+\beta-\gamma)
\end{gathered}
$$

This completes the proof of theorem 3.3.

\section{Starlikeness and Convexity Conditions}

Definition 4.1

A domain $\mathrm{D}$ is called a starlike domain with respect to $w_{0} \in D$ if any line from $\mathrm{w}_{0}$ to any point $w \in D$ lies entirely in $D \cdot[10]$

Definition 4.2

A domain $\mathrm{D}$ is called a convex domain if for any two points $\mathrm{w}_{1}$ and $\mathrm{w}_{2}$ in $D$, the line segment joining $\mathrm{w}_{1}$ and $\mathrm{w}_{2}$ is entirely lying in $D \cdot[10]$

Definition 4.3 
A function $f(z)$ in $\mathrm{S}$ which maps a unit disk to a starlike domain is called a starlike function. [10]

\section{Definition 4.4}

A function $f(z)$ in $S$ which maps a unit $\operatorname{disk} U$ to a convex domain is called a convex function .[10]

Starlikeness and convexity for several subclasses of the class $\mathrm{S}$ have been investigated by many authors. ([10, 14])

A necessary and a sufficient condition for a function $f(z)$ in $\mathrm{S}$ to be starlike is that

$$
\begin{gathered}
f(z)=z^{p}+\sum_{k=1}^{\infty} a_{p+k} z^{p+k}(p \in N=\{1,2,3, \ldots\}) \\
D^{0} f(z)=f(z) \\
D^{1} f(z)=z f^{\prime}(z)=p z^{p}+\sum_{k=1}^{\infty} p+k a_{p+k} z^{p+k} \\
D^{n} f(z)=D\left[D^{n-1} f(z)\right]=p^{n} z^{p}+\sum_{k=1}^{\infty}(p+k)^{n} a_{p+k} z^{p+k} \\
D^{n+1} f(z)=D\left[D^{n} f(z)\right]=p^{n+1} z^{p}+\sum_{k=1}^{\infty}(p+k)^{n+1} a_{p+k} z^{p+k}
\end{gathered}
$$

For starlikeness we have

$$
\begin{gathered}
\operatorname{Re}\left\{\frac{z f^{\prime}(z)}{f(z)}\right\}>0 \\
f^{\prime}(z)=p z^{p-1}+\sum_{k=1}^{\infty} p+k a_{p+k} z^{p+k-1} \\
z f^{\prime}(z)=p z^{p-1+1}+\sum_{k=1}^{\infty} p+k a_{p+k} z^{p+k-1+1}=p z^{p}+\sum_{k=1}^{\infty} p+k a_{p+k} z^{p+k} \\
\operatorname{Re}\left\{\frac{z f^{\prime}(z)}{f(z)}\right\}=\frac{p z^{p}+\sum_{k=1}^{\infty} p+k a_{p+k} z^{p+k}}{f^{p} a_{p+k} z^{p+k}} \\
=\frac{z^{p}+\sum_{k=1}^{\infty} a_{p+k} z^{p+k}}{p}
\end{gathered}
$$

For convexity we have

$$
\operatorname{Re}\left\{1+\frac{z f^{\prime \prime}(z)}{f^{\prime}(z)}\right\}>0
$$




$$
\begin{aligned}
& f^{\prime \prime}(z)=p(p-1) z^{p-2}+\sum_{k=1}^{\infty}(p+k)(p+k-1) a_{p+k} z^{p+k-2} \\
& z f^{\prime \prime}(z)=p(p-1) z^{p-2+1}+\sum_{k=1}^{\infty}(p+k)(p+k-1) a_{p+k} z^{p+k-2+1} \\
& =p(p-1) z^{p-1}+\sum_{k=1}^{\infty}(p+k)(p+k-1) a_{p+k} z^{p+k-1} \\
& f^{\prime}(z)=p z^{p-1}+\sum_{k=1}^{\infty} p+k a_{p+k} z^{p+k-1} \\
& \operatorname{Re}\left\{1+\frac{z f^{\prime \prime}(z)}{f^{\prime}(z)}\right\}=1+\frac{p(p-1) z^{p-1}+\sum_{k=1}^{\infty}(p+k)(p+k-1) a_{p+k} z^{p+k-1}}{p z^{p-1}+\sum_{k=1}^{\infty} p+k a_{p+k} z^{p+k-1}}>0 \\
& =\frac{p z^{p-1}+\sum_{k=1}^{\infty} p+k a_{p+k} z^{p+k-1}+p(p-1) z^{p-1}+\sum_{k=1}^{\infty}(p+k)(p+k-1) a_{p+k} z^{p+k-1}}{p z^{p-1}+\sum_{k=1}^{\infty} p+k a_{p+k} z^{p+k-1}} \\
& =\frac{[p+p(p-1)] z^{p-1}+\sum_{k=1}^{\infty} p+k a_{p+k} z^{p+k-1}+\sum_{k=1}^{\infty}(p+k)(p+k-1) a_{p+k} z^{p+k-1}}{p z^{p-1}+\sum_{k=1}^{\infty} p+k a_{p+k} z^{p+k-1}} \\
& =\frac{\left[p+p^{2}-p\right] z^{p-1}+\sum_{k=1}^{\infty}(p+k) a_{p+k} z^{p+k-1}+\sum_{k=1}^{\infty}(p+k)(p+k-1) a_{p+k} z^{p+k-1}}{p z^{p-1}+\sum_{k=1}^{\infty} p+k a_{p+k} z^{p+k-1}} \\
& \operatorname{Re}\left\{1+\frac{z f^{\prime \prime}(z)}{f^{\prime}(z)}\right\}=\frac{p^{2} z^{p-1}+\sum_{k=1}^{\infty}(p+k) a_{p+k} z^{p+k-1}+\sum_{k=1}^{\infty}(p+k)(p+k-1) a_{p+k} z^{p+k-1}}{p z^{p-1}+\sum_{k=1}^{\infty}(p+k) a_{p+k} z^{p+k-1}}>0
\end{aligned}
$$

\section{Hadamard Product}

Next we define the modified Hadamard product of functions f and g which are defined by (58) and (59) respectively by

$$
f^{*} g(z)=z^{p}-\sum_{k=1}^{\infty} a_{p+k} b_{p+k} z^{p+k}\left(a_{p+k} \geq 0, b_{p+k} \geq 0\right)
$$

Theorem 5.1

If each of the functions $\mathrm{f}$ and $\mathrm{g}$ is in the class $R_{p}^{n}(\alpha, \beta, \gamma, \mu)$ then $f^{*} g(z) \in R_{p}^{n}(\alpha, \beta, \gamma, \eta)$ where 


$$
\eta \geq \frac{\mu^{2}(\alpha p+\beta-\gamma)}{(p+k)[1+(p+k-1) \lambda]^{n}(1+\mu \alpha)^{2}-\mu^{2} \alpha(\alpha p+\beta-\gamma)}
$$

Proof

From theorem 2.1 we have

$$
\sum_{k=1}^{\infty}(p+k)[1+(p+k-1) \lambda]^{n}(1+\mu \alpha) a_{p+k} \leq \mu(\alpha p+\beta-\gamma)
$$

Dividing both sides by $\mu(\alpha p+\beta-\gamma)$ we have

$$
\frac{\sum_{k=1}^{\infty}(p+k)[1+(p+k-1) \lambda]^{n}(1+\mu \alpha) a_{p+k}}{\mu(\alpha p+\beta-\gamma)} \leq \frac{\mu(\alpha p+\beta-\gamma)}{\mu(\alpha p+\beta-\gamma)}
$$

So

$$
\frac{\sum_{k=1}^{\infty}(p+k)[1+(p+k-1) \lambda]^{n}(1+\mu \alpha) a_{p+k}}{\mu(\alpha p+\beta-\gamma)} \leq 1
$$

Again

$$
\begin{gathered}
\frac{\sum_{k=1}^{\infty}(p+k)[1+(p+k-1) \lambda]^{n}(1+\mu \alpha) b_{p+k}}{\mu(\alpha p+\beta-\gamma)} \leq \frac{\mu(\alpha p+\beta-\gamma)}{\mu(\alpha p+\beta-\gamma)} \\
\frac{\sum_{k=1}^{\infty}(p+k)[1+(p+k-1) \lambda]^{n}(1+\mu \alpha) b_{p+k}}{\mu(\alpha p+\beta-\gamma)} \leq 1
\end{gathered}
$$

We need to find the smallest $\eta_{\text {such that }}$

$$
\frac{\sum_{k=1}^{\infty}(p+k)[1+(p+k-1) \lambda]^{n}(1+\eta \alpha)}{\eta(\alpha p+\beta-\gamma)} a_{p+k} b_{p+k} \leq 1
$$

From (72) and (73) we find by means of the Cauchy-Schwarz inequality that

$$
\frac{\sum_{k=1}^{\infty}(p+k)[1+(p+k-1) \lambda]^{n}(1+\mu \alpha)}{\mu(\alpha p+\beta-\gamma)} \sqrt{a_{p+k} b_{p+k}} \leq 1
$$

Thus, it is enough to show that

$$
\begin{gathered}
\frac{(p+k)[1+(p+k-1) \lambda]^{n}(1+\eta \alpha)}{\eta(\alpha p+\beta-\gamma)} a_{p+k} b_{p+k} \leq \frac{(p+k)[1+(p+k-1) \lambda]^{n}(1+\mu \alpha)}{\mu(\alpha p+\beta-\gamma)} \sqrt{a_{p+k} b_{p+k}} \\
\frac{(p+k)[1+(p+k-1) \lambda]^{n}(1+\eta \alpha) \sqrt{a_{p+k} b_{p+k}} \cdot \sqrt{a_{p+k} b_{p+k}}}{\eta(\alpha p+\beta-\gamma)} \leq \frac{(p+k)[1+(p+k-1) \lambda]^{n}(1+\mu \alpha)}{\mu(\alpha p+\beta-\gamma)} \sqrt{a_{p+k} b_{p+k}}
\end{gathered}
$$




$$
\begin{gathered}
(p+k)[1+(p+k-1) \lambda]^{n}(1+\eta \alpha) \mu(\alpha p+\beta-\gamma) \sqrt{a_{p+k} b_{p+k}} \cdot \sqrt{a_{p+k} b_{p+k}} \leq \\
(p+k)[1+(p+k-1) \lambda]^{n}(1+\mu \alpha) \eta(\alpha p+\beta-\gamma) \sqrt{a_{p+k} b_{p+k}} \\
\sqrt{a_{p+k} b_{p+k}} \leq \frac{(p+k)[1+(p+k-1) \lambda]^{n}(1+\mu \alpha) \eta(\alpha p+\beta-\gamma) \sqrt{a_{p+k} b_{p+k}}}{(p+k)[1+(p+k-1) \lambda]^{n}(1+\eta \alpha) \sqrt{a_{k} b_{k}} \cdot \mu(\alpha p+\beta-\gamma)} \sqrt{a_{p+k} b_{p+k}} \leq \frac{\eta(1+\mu \alpha)}{\mu(1+\eta \alpha) .}
\end{gathered}
$$

On the other hand from (75) we have

$$
\begin{gathered}
(p+k)[1+(p+k-1) \lambda]^{n}(1+\mu \alpha) \sqrt{a_{p+k} b_{p+k}} \leq \mu(\alpha p+\beta-\gamma) \\
\sqrt{a_{p+k} b_{p+k}} \leq \frac{\mu(\alpha p+\beta-\gamma)}{(p+k)[1+(p+k-1) \lambda]^{n}(1+\mu \alpha)}
\end{gathered}
$$

Therefore, from (76) and (77) we have

$$
\frac{\mu(\alpha p+\beta-\gamma)}{(p+k)[1+(p+k-1) \lambda]^{n}(1+\mu \alpha)} \leq \frac{\eta(1+\mu \alpha)}{\mu(1+\eta \alpha)}
$$

Which simplifies to

$$
\begin{gathered}
\eta(p+k)[1+(p+k-1) \lambda]^{n}(1+\mu \alpha)(1+\mu \alpha) \leq \mu^{2}(\alpha p+\beta-\gamma)(1+\eta \alpha) \\
\eta(1+\mu \alpha)^{2}(p+k)[1+(p+k-1) \lambda]^{n} \geq \mu^{2}(\alpha p+\beta-\gamma)+\eta \alpha\left(\mu^{2}(\alpha p+\beta-\gamma)\right) \\
{\left[\eta(1+\mu \alpha)^{2}(p+k)[1+(p+k-1) \lambda]^{n}\right]-\left[\eta \alpha\left(\mu^{2}(\alpha p+\beta-\gamma)\right)\right] \geq \mu^{2}(\alpha p+\beta-\gamma)} \\
\left.\eta\left[(1+\mu \alpha)^{2}(p+k)[1+(p+k-1) \lambda]^{n}\right]-\alpha\left(\mu^{2}(\alpha p+\beta-\gamma)\right)\right] \geq \mu^{2}(\alpha p+\beta-\gamma) \\
\eta \geq \frac{\mu^{2}(\alpha p+\beta-\gamma)}{\left.\left[(1+\mu \alpha)^{2}(p+k)[1+(p+k-1) \lambda]^{n}\right]-\alpha\left(\mu^{2}(\alpha p+\beta-\gamma)\right)\right]}
\end{gathered}
$$

\section{Conclusion}

This paper considered review of related literature on Salagean, Al-Oboudi and Opoola differential operators for univalent functions. It also considered literature review for areas related to these three differential operators. Salagean differential operator for $\mathrm{p}$-valent functions was also reviewed. The purpose of there search which is to study some properties of p-valent functions defined by the Al-Oboudi differential operator was highlighted and new subclasses of $\mathrm{p}$-valent functions were defined using Al-Oboudi differential operator. The concept of subordination was used to investigate certain properties of the newly defined subclasses. Theorems were used to establish certain conditions of the newly defined subclasses. These conditions include coefficient inequalities, starlikeness and convexity conditions. Finally, Hadamard product and certain properties of neighbourhoods of the new subclasses of p-valent functions were considered.

\section{References}

[1] Al - Oboudi, F. M. and Haidan, M. M. (2000), Spiralike Functions of Complex Order. J. Nat. Geom. Vol. 19. pp. $53-72$.
[2] Al - Oboudi, F. M. (2004), On Univalent Functions Defined by a Generalized Salagean Operator. International Journal of Mathematics and Mathematical Sciences. Vol. 27. pp. $1429-$ 1436 .

[3] Aouf, M. K., Hossen, H. M., Srivastava, H. M. (2000), Some Families of Multivalent Functions. Applied Mathematics and Computation. Vol. 39. pp. $39-48$

[4] Aouf, M. K., Al - Oboudi, F. M. and Haidan, M. M. (2005), On some Results for $\lambda$-Spiralike and $\lambda$-Robertson Functions of Complex Order. Publ. Instit. Math. Belgrade. Vol. 77. pp. 93 - 98.

[5] Babalola, K. O. and Opoola, P. O. (2006) Integrated Integral Transform of Caratheodory Functions and their Applications to Analytic andUnivalent Functions Tamkang J. Math. 37(4) $355-366$

[6] Bulut, S. (2010), On a Class of Analytic and Multivalent Functions with Negative Coefficients Defined by Al-Oboudi Differential Operator. Studia Univ. "Babes - Bolyai" Mathematica. Vol. LV. No. 4. pp. 115 - 130

[7] Dileep, L. and Latha, S. (2012) On P - valent Functions of Complex Order. Demonstratio Mathematica. Vol. XLV. No. 3 pp. $541-547$.

[8] Fadipe Joseph, O. A. and Opoola, T. O. (2010), On P-valent Functions. ICASTOR. Journal of Mathematical Sciences. Vol. 4. No. 1. pp. $83-86$. 
[9] Fadipe Joseph, O. A. Moses, B. O. and Opoola, T. O. (2015), Multivalence of the Bessel Functions. IEJPAM. Vol. 9. No. 2. pp. $95-104$.

[10] Mahzoon, H. (2011), On Certain Subclasses of Analytic Functions Defined by Differential Subordination. International Journal of Mathematics and Mathematical Sciences. Vol. 2011. pp. $1-10$.

[11] Makinde, D. O. and Opoola, T. O. (2013) On Starlikeness and Convexity Properties of certain Subclass of Funtions. International Journal of Scientific and Research Publication 3(4) $1-3$.

[12] Mostafa, A. O. and Aouf, M. K. (2009) Neighbourhoods of Certain P - valent Analytic Functions with Complex Order. Computers and Mathematics with Applications. Vol. 58. pp. $1183-1189$.

[13] Noor, K. I. (2005), On Classes of Analytic Functions Defined by Convolution with Incomplete Beta Functions. J. Math. Anal. Appl Vol. 307. pp. 339 - 349.

[14] Noor, K. I., Mustafa, S., Malik, B. (2009), On some classes of $\mathrm{P}$ - valently functions Involving certain Carlson Shaffer
Operator. Applied Mathematics and Computation. Vol. 214. pp. $336-341$.

[15] Noor, K. I., Bukhari, S. Z. H., Arif, M. and Nazir, M. (2013), Some Propertiesof $\mathrm{P}$ - valent functions Involving Cho - Kwon - SrivastavaIntegral Operator. Journal of Classical Analysis. Vol. 3. No. 1. pp. $35-43$.

[16] Opoola, T. O. (2017) On a Subclass of Univalent Functions defined by a Generalized Differential Operator. International Journal of Mathematical Analysis. Vol. 11. No. 18. pp. $869-$ 876.

[17] Oyekan, E. A. and Opoola, T. O. (2014), On Subordination for Analytic Functions defined by Convolution. Annales Universitatis Marie Curie - Skiodowska Lubli - Poloma. Vol. LXVIII. No. 1. pp. $1-8$.

[18] Salagean, G. S. (1983), Subclass of Univalent Functions. Lect. Notes. Math. (Springer - Verlag). 1013. 362 - 372.

[19] Sezgin, Akbulut, Ekrem, Kadioglu, and Murat, Ozdemir (2004), On the Subclass of P - valently functions. Applied Mathematics and Computation. Vol. 147. pp. 89 - 96. 